East African Medical Journal Vol. 86 (Supplement) December 2009

DISSEMINATED HISTOPLASMOSIS DIAGNOSED ON BONE MARROW ASPIRATE CYTOLOGY: REPORT OF FOUR CASES

R. Pamnani, MBBS, DCP(Clin. Path.), MD, (Path), Lecturer, J.A. Rajab, MBChB, MMed (Path), MPH, Lecturer, J. Githang'a, MBChB, MMed (Path), Senior Lecturer, Unit of Haematology and Blood Transfusion, Department of Human Pathology and R. Kasmani, MBChB, Registrar, Department of Internal Medicine, School of Medicine, University of Nairobi, P. O. Box 19676 - 00202, Nairobi, Kenya

Request for reprints to: Dr. J. A. Rajab, P.O. Box 19676-00202, Nairobi, Kenya

\title{
DISSEMINATED HISTOPLASMOSIS DIAGNOSED ON BONE MARROW ASPIRATE CYTOLOGY: REPORT OF FOUR CASES
}

\author{
R. PAMNANI, J. A. RAJAB, J. GITHANG'A and R. KASMANI
}

\begin{abstract}
SUMMARY
Histoplasmosis, caused by two varieties of dimorphic fungi, Histoplasma capsulatum variant capsulatum and Histoplasma capsulatum variant duboisii is a systemic fungal infection. It has a worldwide distribution and is shown to be more prevalent in North America and Central America. Both variants occur in Africa. Disease spectrum ranges from asymptomatic primary infection to disseminated disease in immunocompromised patients. Since the upsurge of Acquired Immune Deficiency Syndrome (AIDS) and despite the availability of High Active Anti-Retroviral Therapy (HAART) many patients still present with opportunistic infections of which histoplasmosis is one.

Four cases are presented; two infants and two adults. All had disseminated disease with multiple organ involvement and the disease was not suspected clinically. Diagnosis was made incidentally on bone marrow aspirate cytology. The two adult cases were HIV positive, one with CD4 counts of 132 cells/microlitre and was not on HAART. The other was on HAART but the CD4 had not been determined. One of the infants tested HIV negative and the others status was unknown. A high index of suspicion is required for diagnosis as the disease may mimic tuberculosis(TB) and other causes of hepatosplenomegaly such as visceral leishmaniasis.

Laboratory diagnosis includes culture, direct staining, antigen and antibody detection. Antibody detection may give false negative in the immunocompromised patient. The infection responds well to antifungal agents (amphotericin B is the drug of choice) and life long maintenance therapy may be required in AIDS especially if CD4 counts remain less than 150 cells/microlitre.

Histoplasmosis should be a differential diagnosis in immunosuppressed patients with unexplained fever, weight loss, hepatosplenomegaly and chest findings especially if not responding to anti-TB treatment.
\end{abstract}

\section{INTRODUCTION}

Histoplasmosis is an endemic infection in most of the United States and can be found worldwide. The spectrum of disease ranges from asymptomatic infection to severe disseminated disease. Life threatening illness is usually associated with an immunocompromised state. Bone marrow, liver, skin, and mucocutanoeus lesions usually reveal organisms which may also be visualised on routine peripheral blood smears in patients with disseminated disease. Four patients all diagnosed incidentally on bone marrow aspirate cytology are presented. Available laboratory diagnostic methods and treatmentoptions are reviewed.

\section{CASE REPORTS}

Case 1: A six year old female from Machakos (Eastern province) with fever, cough and abdominal pain for five months, and had showed no response to treatment for tuberculosis and leishmaniasis. He was ill looking, pale, wasted, febrile with bilateral pitting oedema of the legs, hepatosplenomegaly, scattered bilateral basal crepitations on chest examination. There was nolymph nodeenlargement. Laboratory findings showed $\mathrm{Hb}-7.8$ gm/ dl,WBC-7.7x109/ L(N-51,L-41,M-8), platelets-200 x $10^{9} / \mathrm{L}$, ESR-47 mm/1st hr. Liver function tests were withinnormal range(Total protein-58g/L(Albumin-23, Globulin-35gm/L), Sputum was negative for AcidFast Bacilli(AFB), HIV-negative, chestX-ray showed patchy 
pneumonic infiltrate. Abdominal ultrasonography confirmed hepatosplenomegaly.

Case 2: A one year nine months old female from Tana River District (Coast province) presented with anorexia, abdominal swelling and passing black coloured stools for two months. She was found to be in fairgeneral condition, pale, with hepatosplenomegaly. There was nolymph node enlargement. Adifferential diagnosis of malarial splenomegaly syndrome and visceral leishmaniasis was made. Laboratory findings showed-Hb-7.6 gm/ dl,WBC- $4.6 \times 10^{9} / \mathrm{L}$ (N-34, L-61, M-5), platelets of $-114 \times 10^{9} / \mathrm{L}$. Blood slide for malaria parasite was negative. Stool for ova and cysts was also negative. Urine examination showed no significant finding. The mother declined to consent for HIV testing. Abdominal ultrasonography confirmed the hepatosplenomegaly.

Case 3: A 49 year old male from Kitui (Eastern province), known HIV positive for three years, who presented with fever, abdominal swelling, lower limb swelling for five months, night sweats, weight loss, anorexia, malaise and body aches. He was found to be wasted, febrile, pale, with cervical and inguinal lymphnode enlargement, bilateral pitting pedal oedema and hepatosplenomegaly. Laboratory findings were $\mathrm{Hb}-5.2 \mathrm{gm} / \mathrm{dl}$,WBC- $5.1 \times 10^{9} / \mathrm{L}$, platelets $6 \times 10^{9} / \mathrm{L}$. Renal function tests were within normal range. Liver function tests showed protein of $53 \mathrm{gm} / \mathrm{L}$ (Albumin- $18 \mathrm{gm} / \mathrm{L}$ ),AST- 94, ALT- 30, ALP- 214, GGT- 62, hepatitis B and C screening was negative. CD4 counts were 132 cells / microlitre. Chest $\mathrm{X}$-ray was normal and abdominal ultrasonography showed hepatosplenomegaly.

Case 4: A 50 year old female from central province, known HIV positive for six months on HAART, treated for TB a month previously presented with cough with blood stained sputum, oral ulcers, lower limb weakness for a month, chills, night sweats and anorexia. She was found to be sick looking, febrile wasted, drowsy with pallor and bilateral pedal oedema. She had submental, jugulo-digastric lymphnode enlargement febrile. There were multiple skin lesions on the trunk and extremities. There were left upper chest crepitations, hepatosplenomegaly and reduced muscle bulk globally with hypereflexia in the upper limbs. Laboratory findings showed $\mathrm{Hb}-5.58 \mathrm{gm} / \mathrm{dl}$,WBC- $3.6 \times 10^{9} / \mathrm{L}$ ( N- 80, L- 11, M9) and platelets of $119 \times 10^{9} / \mathrm{L}$, urea, electrolytes and creatinine were within normal range. Liver function tests showed protein- $43 \mathrm{gm} / \mathrm{L}$, albumin $17 \mathrm{gm} / \mathrm{L}$, AST- 76, ALT-14, ALP-423, GGT- 102. Hepatitis B and $\mathrm{C}$ screening were negative.

ChestX-ray showed leftupperlobeconsolidation. CT scan of the brain was normal and abdominal ultrasonography confirmed hepatosplenomegaly.
All the patients were started on antifungal agents (fluconazole or cotrimoxazole) along with supportive therapy. One patient(case 2) responded positively to treatment while two (case 1 and 4 ) succumbed to the disease and one(case 3) was lost to follow up.

\section{DISCUSSION}

Disseminated histoplasmosisis an AIDSdefiningillness caused by a dimorphic fungi Histoplasma capsulatum variant capsulatum, the other variant Histoplasma capsulatum variant dubosii which causes African histoplasmosis (1) is endemic in Central and West Africa and is rarely associated with HIV infections (2). Before the era of HAART, the prevalence of Histoplasma capsulatum variant capsulatum in HIV - infected patients in hyperendemic areas of South Eastern USA reached up to $30 \%$ (3). Other patient groups at risk for disseminated histoplasmosis include transplant recipients, those with haematologic malignancies and those on steroids (4). Infants may also develop severe life threatening infections. Two patients who had HIV infection in this report one had been on HAART for more than three months and one had CD4 counts of 132 cells / microlitre. Patients with CD 4 counts of $<150$ cells/ul are most at risk. Symptoms of disseminated histoplasmosis include fever, malaise, anorexia and weight loss which were present in all the four reported cases. Hepatosplenomegaly and lymphadenopathy are also common findings. Anaemia and petechiae may be a finding if pancytopaenia is present.

Only one of the patients had mucous membrane ulcerations as well as skin ulcers, nodules or mollusum like papules that are reported in some patients. In infants and patients with AIDS, a rapidly fatal acute form of disseminated severe disease can present as sepsis syndrome with hypertension, disseminated intravascular coagulation, renal failure and acute respiratory distress (4). In this report one adult patient succumbed acutely to the disease and one infant died after six months history of illness.

In a series of 164 patients with HIV and disseminated histoplasmosis Elizabeth et al (5) in Brazil showed the most frequent manifestations to be fever $(95 \%)$, cough $(75 \%)$, weight loss $(72 \%)$, diarrhoea $(61 \%)$ and adynamia (56\%).

Disseminated histoplasmosis may involve any organ including; Gastrointestinal tract (colon, small bowel with ulcerations, strictures and sometimes perforations); adrenal gland (patients may present with Addison's disease) tongue, gingivae, buccal mucosa, lips, pharynx and larynx are also commonly affected than in any other endemic mycoses (4). Symptomatic disease is rare in the genitourinary tract, kidney and osteoarticularsites. Other raresitesinclude; Gallbladder, breast, thymus and thyroid gland and are usually discovered at autopsy. Other rare manifestations of disseminated histoplasmosisinclude; endocarditis and 
vascular infection and central nervous system infection manifesting as meningitis and brain abscesses.

In the immunocompetent persons exposed to $H$. capsulatum, symptomatic infection is uncommon and most patients have nosymptoms, the disease spectrum varies from (i) Acute pulmonary histoplasmosis occurring mostly in children and is accompanied by rheumatologic and dermatologic manifestations. (ii) Chronic cavitary pulmonary histoplasmosis in older patients. Pre-existing chronic obstructive pulmonary disease, especially emphysema is usually present. Progressive pulmonary insufficiency usually leads to death. (iii) Granulomatous mediastinitis with massive enlargement of multiple nodes that are matted. Majority of patients are asymptomatic but others may experience pressure symptoms when the enlarged nodes infringe on the superior vena cava, the bronchii or the oesophagus (iv). Mediastinal fibrosis which is uncommon is frequently lethal, results from uncontrolled fibrotic response to infected caseous nodes resulting in the encasement of nodes and vital structures of themediastinum. Most patients areyoung adults between the ages of 20-40 years with a slight predominance of women. Other manifestationsinclude pericarditis, pleural disease and broncholithiasis (4).

Bone marrow examination in disseminated infections, as in all the cases in this report, usually revealsorganisms(Figure1). The reported sensitivity of bone marrow fungal stains in patients with AIDS and histoplasmosis has ranged from $67 \%$ to $100 \%$ (6). These are also seen in liver, skin and mucocutaneous lesions. Routine peripheralblood smears will sometimes show yeasts within neutrophils in patients who are seriously ill. Tissue biopsy usually shows the distinctive $2-4 \mu \mathrm{m}$ oval narrow - based budding yeasts.

Figure 1

Histoplasma capsulatum yeasts in bone marrow macrophages

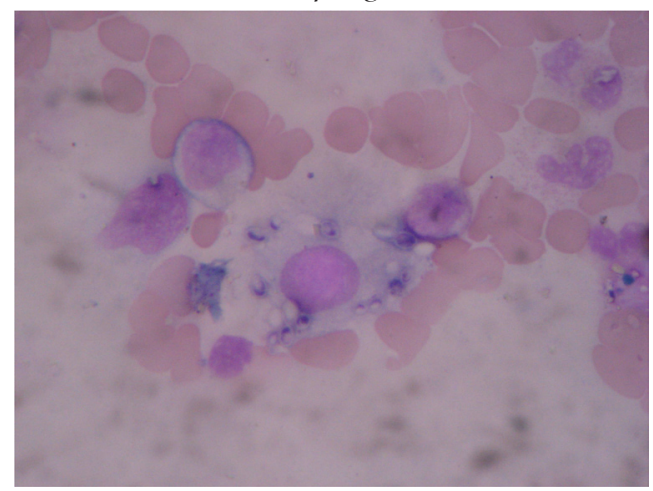

Other organisms that may mimic the appearance of $H$. capsulatum include; Leishmania (as was the case with of one of the patients in this report) and Candida. Tissues are stained with methenamine silver or periodic acid schiff (PAS) stains. Yeasts are usually found within macrophages but can also be seen free in tissues.
Other diagnostic methods include culture of tissue or body fluids on Sabourand's dextrose agar. Growth can take up to six weeks. Definitive identification of $H$. capsulatum from other mycosis is made by a chemiluminescent DNA probes.

Antigen detection from urine or serum was first described in 1986 using radio immunoassay is now performed by a sandwich enzyme immunoassay. In HIV patients the antigen is detected in urine in $95 \%$ of patients and in $86 \%$ of patients in serum (7). Connolly et al (8) reported $100 \%$ antigen detection in urine and $92 \%$ detection in serum. False positive reactions may occur in other endemic mycoses. Work on PCR assays is currently ongoing.

Antibody assays by complement fixation and immmunodiffusion assay are useful in patients who have chronic forms of histoplasmosis. False positive results are seen in patients with lymphoma, TB, sarcoidosis and other fungal infections. Assays are less useful in immunossupresion as these patients mount a poor response. Skin reagents are no longer commercially available. Skin tests historically are essential in defining areas where $H$. capsulatum is endemic but are not very helpful as a diagnostic test due to cross reactions with other fungi.

Amphoterecin B remained the drug of choice until 1990s when azoles were introduced. Mortality in those who had disseminated disease and not treated was $83 \%$ compared to $23 \%$ in amphotericin $\mathrm{B}$ treated patients. Introduction of ketoconazole enabled treatment in the out-patient setting with oral medication. Treatment is taken for at least six months. Other choices include itraconazole which has fewer side effects and has proved effective in AIDS patients, other newer azoles that have been tried include voriconazole and posaconazole (4).

The length of therapy depends on severity of the infection and immune status. Some guidelines recommend $6-18$ months in total because of high rate of relapse for AIDS patients. Maintenance azole therapy is continued for life after initial response to antifungal therapy especially if CD4 counts remain below 150cells / $\mu$ l. With the use of effective HAART, rates of infection have fallen in the AIDS population and prophylaxis is no longer recommended (9).

In summary, disseminated histoplasmosis should be suspected in any immunocompromised patient presenting with unexplained fever especially if severely ill. A high index of suspicion is required as it may mimic other illness especially tuberculosis, leishmaniasis and typhoid. Bone marrow examination even with routine stains is a useful diagnostic test when cultures and serological tests are unavailable.

\section{ACKNOWLEDGEMENT}

To Kenyatta National Hospital wards 7 and 8 and to Getrude's Garden Children's Hospital. 


\section{REFERENCES}

1. H.C Gugnani. Histoplasmosis in Africa: A review Indian J. Chest Dis. Allied Sci. 2000; 42: 271-277

2. P. Loulergue, F. Bastides, V. Bavdovin, et al. Emerging Infectious Disease wwww.cdc.gov/aid.Vol. 13, No. 11. November 2007.

3. Mckinsey, D.S., Spiegel, R.A., Hutwagner, L., et al. Prospective study of histoplasmosis in patients infected with human immunodeficiency virus; Incidence risk factors and pathophysiology. Clin. Infect. Dis. 1997; 24: 1195-2203.

4. Kauffman, C. Histoplasmosis: a clinical and laboratory update. Clinical Microbiol. Rev. 2007; 20: 115-132.

5. Elizabeth, F., Daher, Geraldo, B., Silva Jr, Fernando, A.S., et al. Clinical and laboratory features of disseminated hispoplasmosis in HIV patients from Brazil. Trop. Med. Inter. Hlth. 2007; 12: $1108-1115$.
6. Neubauer,M.A. and Bodensteiner,D.C.Disseminated histoplasmosis in patients with AIDS. South Med. J. 1992; 85: 1116-1170.

7. Wheat, L.J. and C.A. Kauffman. Histoplasmosis. 2003 Infect. Dis. Clin. N. Am. 17:1-19.

8. Connolly,P.A., Durkin, M.M., Lemonte, A.N., et al . Detection of histoplasma antigen by a quantitative enzymes immunoassay. Clin. Vaccine Immunol. 2007; 14: 1587-1591.

9. McKinsey,D.S., L.J. Wheat, G.A., Cloud, M., et al. Itraconazole prophylaxis for fungal infections in patients with advanced human immunodeficiency virus infection: randomized, placebo-controlled, double-blind study. Clin. Infect. Dis. 1999; 28: 1049-1056. 